was huge, the Sichuan earthquake alone resulted in an economic loss of 845.1 billion Chinese Yuan. However, psychosocial factors did not receive attention by Chinese Government and academics. Conclusions: The characteristics and impact of disasters should be analyzed to scientifically provide useful information for natural disaster mitigation in China.

Prehosp Disaster Med 2011;26(Suppl. 1):s126-s127

doi:10.1017/S1049023X11004183

(P1-87) Preparedness of Healthcare Facilities for an Influenza Pandemic - Protecting the Healthcare Workers A.L. Hollingworth

Respiratory, Darlinghurst, Australia

Aim: To assess the preparedness of hospitals with respect to protecting health care workers (HCWs) during a pandemic.

Methods: A self-administered questionnaire was performed between November 2009 and January 2010, and a scoring system was developed to provide a quantifiable measure of preparedness.

Results: A total of 12 hospitals in NSW, Australia, were approached - six regional hospitals ( $\mathrm{RH}$ s) and six tertiary referral centres (TRCs). The study was extended to assess three hospitals in England, allowing a limited comparison between the hospitals in Australia that had faced the initial wave of the H1N1 ("swine flu") pandemic and the hospitals in the UK that had more time to prepare for the outbreak. Response rates were $66 \%$ from the TRCs, $33 \%$ from the RHs, and $100 \%$ from the English hospitals. The overall preparedness scores were relatively high, with a median TOTAL score (adjusted) of 50.75 out of 70 . The demographic that scored the highest Total was tertiary referral centres in Sydney. All English hospitals scored below the median. However, the range of scores across hospitals was quite narrow (45.1 - 57.1 adjusted). Scores were generally high for the areas of Preparedness, Infection control, Education and Training. Scores for Vaccination were more variable. The category that consistently demonstrated the lowest scores was that of Psychosocial Welfare and Assistance, despite this being found in previous research to be an integral part of that which HCWs have identified as important.

Conclusions: Given their integral role in pandemic response, protecting HCWs must be a priority as part of any pandemic preparedness plan. This goes beyond protection from infection, extending into aspects of physical and psychological wellbeing. Identifying these issues and addressing them is the key to maximising staff support and morale, and minimising staff absenteeism at such a crucial time.

Prehosp Disaster Med 2011;26(Suppl. 1):s127

doi:10.1017/S1049023X11004195

(P1-88) Development of a First Hospital Based Trauma Registry at JPN Apex Trauma Center, India

A. Gupta, S. Kumar, S. Sagar, M. Singhal, B. Mishra, M.C.

Misra

JPN Apex Trauma Center, New Delhi, India

Although Injury is being looked into as a major public health problem in India, most of the data coming is mortality related data from the National Crime Records Bureau and projections based on that data. There is complete absence if injury related data both surveillance data as well as outcome based data. Apex Trauma Center, All India Institute of Medical Sciences, New Delhi is one of the pioneering centers to understand the need to record the injury related data of all trauma cases which are admitted to the Apex Center, thus establishing a first of its kind hospital based Trauma Registry in India. This trauma registry will serve as a means for collating trauma data that will further help in the evaluation, prevention, and research of trauma care and can be used for quality control and planning future research and injury prevention activities, in India. Later, the center has an objective of networking all regional hospitals for data collection with an aim to establish a National Trauma Registry. Although several trauma registry software's exist from Western hemisphere but the Apex Trauma Center decided to formulate and designed its own Trauma Registry form and develop the related software which includes: Basic Identification; Demographic profile; Brought by personnel and vehicle; Condition at time of arrival; ED Interventions; Detailed Diagnosis; Definitive Procedures; Disposition/ Outcome The Trauma registry is being maintained, under the leadership of a Faculty and the data is collected and entered by the Trauma Nurse Coordinators, who follow the patient from admission to discharge. The data collection for the JPNATC Trauma Registry had started w.e.f. April 2009, but initially there were usual problems of data loss and non-availability of data. This has been overcome gradually and we hope that the registry will attain its full potential in another year or so.

Prehosp Disaster Med 2011;26(Suppl. 1):s127 doi:10.1017/S1049023X11004201

\section{(P1-89) Psychosocial Tsunami- Financial Crisis} M.C. Saenz

Human Resources, Lomas De Zamora, Argentina

Psychosocial Tsunami Financial Crisis Tragedies produced by nature have patterns similar to psychosocial emergencies. The disruptive effects impact on Public Health. Unemployment covers society and doesn't allow personal aptitudes to emerge and sinks people in hopelessness. There is a perception of constant risk. People are in alert with all the effects of sharp and chronic stress and in some occasions Post Traumatic Stress.

Objective: To get an efficient answer to reality from this impoverished group with severe effects facing working uncertainty and unsatisfied basic needs. To avoid the social tragedy to be a big wave that sinks a big part of the population very quickly. To train people on the importance of work to get a better quality life for each participant, the family and community.

Methodology and Diagnosis: 6200 people were trained in twenty months and motivated to work in a population of 95000 citizens approximately. They got a salary and social security financed by the government and articulated with the NGO. They were organized according to working experience and abilities and a supervisor was elected every ten people. Each participant had been polled to reach these conclusions. Industrial security, health care, and group work abilities were some of the syllabus topics. Some of the tasks performed were: painting, gardening, public places embellishment and fixing, administrative duties, river cleaning, etc. 